\title{
WR galaxies and PAH: observations and modeling
}

\author{
A. Yeghikyan ${ }^{1 *}$, J. Martirosyan ${ }^{2}$ \\ ${ }^{1}$ NAS RA V. Ambartsumian Byurakan Astrophysical Observatory (BAO), Armenia \\ ${ }^{2}$ Yerevan State University, Armenia \\ *E-mail: ayarayeg@gmail.com
}

\begin{abstract}
This report analyzes the archival data of the Spitzer observatory for $9 \mathrm{WR}$ galaxies showing PAH emission lines at 6.2 and $7.7 \mu$. For these galaxies, the effective radii of the emitting regions and the total number of hydrogen ionizing quanta were calculated on the base of the fluxes corrected for interstellar extinction. Using the observed values (which are obtained using SDSS data), and taking as an example stars of the WNh type $\left(T_{s}=50000 \mathrm{~K}, R=20 R_{\odot}\right)$, the number of massive WR stars in the cluster and the distance to the nearest neighbor are determined. With these data, the theoretical Cloudy models, in particular, the distribution of $\mathrm{H}$ and $\mathrm{He}, \mathrm{C}, \mathrm{O}, \mathrm{Ne}$ atoms and ions along the radius are calculated. The theoretical intensities of the characteristic PAH bands at 6.2 and $7.7 \mu$ were obtained for two types of PAH (containing 15 and 120 carbon atoms, respectively). The corpuscular radiation doses accumulated in $\mathrm{PAH}$ molecules due to hard irradiation by $\mathrm{MeV}$ protons were calculated and it was shown that such molecules had to decay for a time less than the lifetime of the cluster. A conclusion is drawn about the permanent source of PAH, most likely, the remnants of supernova explosions.
\end{abstract}

Keywords: WR galaxies - PAH - observations - Cloudy modeling - energetic particles - PAH survivability.

\section{Introduction}

The activity of galaxies with a high star formation rate is characterized by the birth of a large number of stars of large mass in one cluster (Crowther et al. 2005). If the mass of the $\mathrm{O}$ class star exceeds $25 M_{\odot}$ (with the solar abundance of chemical elements), the star evolves into a WR phase of no more than 0.5 Myr lifetime, characterized by a very high mass loss rate (of the order of $10^{-5} M_{\odot} /$ year) and speeds of $500-5000 \mathrm{~km} / \mathrm{s}$, but mostly, $1500-2000 \mathrm{~km} / \mathrm{s}$ (Meynet and Maeder 2005). In this case, the lifetime of 
massive $\mathrm{O}$ stars stage does not exceed 5 million years (at a mass loss rate of about $10^{-6} M_{\odot} / \mathrm{yr}$ ). If the number of very massive stars in one cluster is sufficiently large, then many stars can also be caught in the WR phase at the same time, since the number of stars in the WR phase is comparable to the number $\mathrm{O}$ of stars (Crowther et al. 2005). This circumstance is confirmed in the spectra of many active galaxies with characteristic features of WR stars. First of all, it is a characteristic emission component in the spectrum with a width of about 40-50 Aat a center at $4650 \AA$ (for WN stars) which is a superposition of 3 lines, He II $4686 \AA$, NeIII $4640 \AA$ And CIII/IV $4650 \AA$. A feature of $5808 \AA$ (for WC stars) related to CIV 5808 Ais also possible. With these features, such galaxies are called WR galaxies (Conti 1991), and they are good indicators of the star formation rate, since the lifetime of massive $\mathrm{O}$ stars does not exceed 5 million years (Meynet and Maeder 2005). Upon completion of evolution, they explode in the form of supernovae Ib, c. Perhaps they are also associated with the predecessors of GRB (Vink1 et al. 2011). The active regions of these galaxies, and the WR galaxies themselves, include a morphologically diverse range of objects with an increased star formation rate (SB), for example, giant HII regions in spiral arms, irregular galaxies, blue compact dwarf galaxies, bright galaxies in the process of merging, galaxies with active nuclei, etc. (Brinchmann et al. 2008). The number of massive stars in one (active) cluster is estimated as 100-100000 (Crowther et al. 2005). It is interesting to note that the intensity of the emission "bump" in the spectra of WR galaxies depends on the degree of "metallicity": the lower the metal content, the less intense wind can generate a WR star, since it is the radiation pressure in the resonance lines of the $\mathrm{C}, \mathrm{N}, \mathrm{O}$ ions that accelerates the wind to the observed speeds. However, the "bump" is also observed in objects with a very low "metallicity", for example, in Izw18 (Crowther et al. 2005, Brinchmann et al. 2008), although it requires the presence of very massive stars with a mass more than $80 M_{\odot}$ (with a metallicity of 0.05 ).

In the spectra of many galaxies, including active ones, characteristic bands of many molecules are observed, for example, water, carbon monoxide, polycyclic aromatic hydrocarbons (PAHs), etc. In particular, PAHs are also observed in the spectra of WR galaxies (Martirosyan, Sargsyan 2012), in this case, the emission is provided by PAH molecules with a relative concentration to hydrogen (in all forms) of the order of $2 \cdot 10^{-7}-2 \cdot 10^{-8}$ and a carbon to hydrogen ratio of about $3 \cdot 10^{-4}$ (Ferland et al. 2017). In the theoretical interpretation of the spectra of PAHs, the number of carbon atoms is usually in the range from 15 to 120 (Ferland et al. 2017). PAHs are very sensitive to the intense radiation fields, which makes it possible to use the equivalent widths of $\mathrm{PAH}$ emission lines as an indicator, allowing to distinguish galaxies with active nuclei (PAH lines are very weak or absent) from galaxies with intense star formation (lines are relatively strong) (Martirosyan, Sargsyan 2012). From an evolutionary point of view, the question 
of PAHs sources in active galaxies in general, and in WR galaxies in particular, is very interesting and directly related to the conditions of their survival in the presence of hard electromagnetic and corpuscular radiation. The exact estimation of the UV radiation field depends on many parameters, e.g. on the amount of neutral gas in the cluster, the theoretical value of which is strongly model-dependent, and there is no observational data for WR galaxies (Risaliti et al. 1999). This article is devoted to the calculation of PAH radiation doses by corpuscular radiation from massive stars in a WR galaxy, the problem of the survival of PAH molecules under these conditions, and a discussion on the issue of PAH sources in the such galaxy .

\section{Observational data}

There are $570 \mathrm{WR}$ galaxies, 422 of which are SB (Brinchmann et al. 2008). In (Martirosyan, Sargsyan 2012), 22 WR galaxies were selected from this list, for which Spitzer observations in the region of 5.2-14.5 $\mu$ and 14$38 \mu$ are known, including emission lines of PAHs at 6.2 and $7.7 \mu$. For these galaxies, also known are the SDSS data (sdss.org 2018) on the main emission lines in the visible region, which allowed us to carry out standard diagnostics of the HII regions associated with the WR regions of galaxies. In particular, the electron concentrations and temperatures were determined, as well as the interstellar extinction values for 9 galaxies according to the formulas (Crowther 2007):

$$
\begin{gathered}
\frac{F\left(H_{\alpha}\right)}{F\left(H_{\beta}\right)}=\frac{I\left(H_{\alpha}\right)}{I\left(H_{\beta}\right)} \cdot 10^{-c\left(H_{\beta}\right)\left[x\left(H_{\alpha}\right)-x\left(H_{\beta}\right)\right]} \\
E(B-V)=0.77 \cdot c\left(H_{\beta}\right),\left[x\left(H_{\alpha}\right)-x\left(H_{\beta}\right)\right]=0.346, R_{V}=3.1 \\
\frac{I\left(H_{\alpha}\right)}{I\left(H_{\beta}\right)}=2.86, m=m_{o b s}-A_{V}, A_{V} \approx 3 \cdot E(B-V) \\
F\left(H_{\beta}\right)=3 \cdot 10^{-9} \cdot W\left(H_{\beta}\right) \cdot 10^{-0.4 m}\left(\mathrm{erg} / \mathrm{cm}^{2} / \mathrm{s}\right) \\
L\left(H_{\beta}\right)=4 \pi d^{2} F\left(H_{\beta}\right),(d-\text { distance to the galaxy }) .
\end{gathered}
$$

All initial values of 9 galaxies (from 22 galaxies) are shown in Table 1.

Further, since the data on interstellar extinction are known only for 9 galaxies then we will carry out all calculations only for these objects. So, according to the luminosities in the $H_{\beta}$ line corrected for the interstellar extinction and the calculated values of the emission coefficient in the same line (in the unit volume of the HII region) we have well known relation (with the value of $b_{4}\left(T_{e}\right)$ defined in (Ferland et al. 2017)):

$$
E\left(H_{\beta}\right)=22.4 \cdot 10^{-20} \frac{\left(n_{e}\right)^{2} b_{4}\left(T_{e}\right)}{\left(T_{e}\right)^{3 / 2}} e^{9814 / T_{e}}\left(\mathrm{erg} / \mathrm{cm}^{2} / \mathrm{s}\right)
$$




\begin{tabular}{|c|c|c|c|c|}
\hline WR number & ra & dec & z & EW, PAH 6.2 $\mu, \AA$ \\
\hline 31 & 125305.96 & -031258.94 & 0.02276 & 0.01 \\
\hline 40 & 173501.24 & +570308.55 & 0.04722 & 0.01 \\
\hline 48 & 005527.46 & -002148.76 & 0.16741 & 0.09 \\
\hline 108 & 093813.49 & +542825.03 & 0.10213 & 0.21 \\
\hline 190 & 103410.15 & +580349.06 & 0.00749 & 0.15 \\
\hline 277 & 110508.11 & +444447.24 & 0.02154 & 0.3 \\
\hline 288 & 132844.05 & +435550.51 & 0.02795 & 0.37 \\
\hline 292 & 152238.09 & +333135.81 & 0.12529 & 0.26 \\
\hline 296 & 101624.51 & +375445.96 & 0.00394 & 0.02 \\
\hline
\end{tabular}

Table 1: Observational values of WR galaxies with PAH data

Then one can determine the effective volume of radiation as a ratio

$$
V_{\text {eff }}=\frac{L\left(H_{\beta}\right)}{E\left(H_{\beta}\right)},
$$

and the effective radius

$$
R_{e f f}=\sqrt[3]{V_{e f f}}
$$

Now we can determine the number of ionizing hydrogen quanta by the known formula

$$
\Sigma N\left(L_{c}\right)=q \cdot N\left(H_{\beta}\right),
$$

where $q=10.97$ at $T_{e}=10000 \mathrm{~K}$ and $N\left(H_{\beta}\right)=L\left(H_{\beta}\right) / h \nu\left(H_{\beta}\right) s^{-1}$ - number of quanta in the $H_{\beta}$ line, with the energy of a single quantum equal to $h \nu\left(H_{\beta}\right)=4.09 \cdot 10^{12} \mathrm{erg}$. To estimate the hardness of photon radiation, the parameter $U$ is usually used (Ferland et al. 2017):

$$
U=\frac{\Sigma N\left(L_{c}\right)}{4 \pi c \cdot n_{e} \cdot\left(R_{e f f}\right)^{2}} .
$$

The values of $N\left(L_{c}\right)$ and $U$ are given in Tables 2.

\begin{tabular}{|c|c|c|c|c|c|c|}
\hline WR number & $n_{e}, \mathrm{~cm}^{-3}$ & $T_{e}, \mathbf{K}$ & $\Sigma N\left(L_{c}\right), s^{-1}$ & $N_{s}$ & $\mathbf{R}, \mathbf{p c}$ & $\mathbf{l g}(\mathbf{U})$ \\
\hline 31 & 390.7 & 19830 & $2.310+57$ & $2.310+7$ & $2.199+21$ & 0.5113 \\
\hline 40 & 254.7 & 9581 & $1.942+54$ & 19420 & $3.408+20$ & -0.7588 \\
\hline 48 & 302.7 & 9077 & $1.623+54$ & 16230 & $2.564+20$ & -0.6647 \\
\hline 108 & 60.59 & 15300 & $9.508+53$ & 9508 & $6.331+20$ & -0.9835 \\
\hline 190 & 62.59 & 12490 & $5.812+51$ & 58 & $1.384+20$ & -1.8905 \\
\hline 277 & 80.19 & 15750 & $2.321+53$ & 2321 & $3.268+20$ & -0.1432 \\
\hline 288 & 94.53 & 8708 & $3.034+53$ & 3034 & $3.237+20$ & -1.0899 \\
\hline 292 & 204.3 & 24970 & $5.054+54$ & 50541 & $4.884+20$ & -0.5604 \\
\hline 296 & 15.07 & 10360 & $3.248+51$ & 32 & $3.136+20$ & -2.2354 \\
\hline
\end{tabular}

Table 2: Observed and calculated data for WR galaxies 


\section{Young stellar clusters in WR galaxies}

The number of stars in the cluster can be estimated by the ratio $N_{s}=$ $\Sigma N\left(L_{c}\right) / N\left(L_{c}\right)$, where $\Sigma N\left(L_{c}\right)$ is the total number of quanta ionizing hydrogen in the nebula, and $N\left(L_{c}\right)$ is the number of such photons emitting from one star per unit time. Taking, as an example, WN type stars with the presence of hydrogen $\left(T_{s}=50000 \mathrm{~K}, R_{s}=20 R_{\odot}\right.$, but the presence of hydrogen is not important in this case), we have the amount of such quanta by the relation

$$
N\left(L_{c}\right)=4 \pi R_{s}^{2} \cdot \frac{2 \pi}{c^{2}}\left(\frac{k T_{s}}{h}\right)^{3} \cdot \delta=1.8 \cdot 10^{50} \mathrm{~s}^{-1}
$$

where

$$
\delta=\int_{x_{0}}^{\infty} \frac{x^{2} d x}{e^{x}-1}, x_{0}=\frac{k_{e} T_{e}}{13.6}, k_{e}=8.6 \cdot 10^{-5} \frac{\mathrm{eV}}{\mathrm{K}},
$$

while according to calculations based on stellar atmospheric models, $N\left(L_{c}\right)=$ $1.0 \cdot 10^{-50} \mathrm{~s}^{-1}$ (Crowther 2007; Hamann et al. 2006). So, we get an estimate of the number of massive stars in the cluster, for $9 \mathrm{WR}$ galaxies, for which interstellar extinction data are known. The results are shown in Table 2. With three exceptions, the number of massive stars in the cluster was several thousand or tens of thousands. As cluster sizes from Table 2 are about 100 parsec, these clusters are close to the super-associations according to (Ambartsumian 1964). Being less (in the sense of the classical definition) in size, absolute luminosity and age, and not quite coinciding in the type of host galaxies, these systems with a large number of massive hot and young stars, mostly coincide with the definition of V. Ambartsumyan and are one of the most important star formation activity indicators. So this term also will be used below - superassociation, as a young cluster of a very large number of very massive stars. At present, they are also called Super Star Clusters (Crowther et al. 2005).

Further, Table 3 shows the distances to the nearest neighbor star, calculated for a sphere uniformly filled with similar stars, using the obvious formula

$$
d=\frac{R_{e f f}}{\sqrt[3]{N_{s}}} .
$$

Then, models of the ionization structures of nebulae associated with WR clusters were calculated. The parameters of the model have already taken the distances to the nearest neighbor as the characteristic thickness of the nebula, $n_{H} \approx n_{e}$ as the characteristic concentration of hydrogen, the ionization parameter $U$, the blackbody temperature $T_{s}=50000 \mathrm{~K}$, the effective radius $R_{\text {eff }}$ as the characteristic radius of the incident radiation, chemical composition was assumed to be equal to solar, and the value of the turbulent velocity in the medium about $10 \mathrm{~km} / \mathrm{s}$. It is easy to show on the 
base of calculations of ionizational structures that at a distance of more than $1 \mathrm{pc}$, the medium at such a density becomes neutral and then molecular, protecting the PAH molecules from destruction by UV photons. The same is true for other galaxies from the list. Let us get started, therefore, to estimate of the degree of impact of corpuscular irradiation on PAH molecules, since for energies of the order of $1 \mathrm{MeV}$ and higher, nebulae in WR clusters are transparent for such radiation.

\section{Corpuscular radiation fluxes, radiation doses and survivability of PAH}

There are a large number of analytical and numerical models describing intensities of energetic particles accelerated at the shock that is formed during the interaction of a fast stellar wind with a given nebula (in this case, with a super-association nebula) (Drury 1983; Decourchelle 2008). Detailed calculations take into account the many features of the process, such as the formation of a spectrum of particles, the orientation of the magnetic field vector with respect to the normal to the front, the presence of sources of super-thermal particles experiencing acceleration by the Fermi 1 diffusion mechanism. These theories predict reasonable values of particle intensities, in particular, galactic cosmic rays, and are now generally accepted. For example, in the case of the heliosphere, that is, the shock wave front at the interface of the solar wind interaction with the surrounding interstellar gas, the intensities of the accelerated particles are in good agreement with direct observations of the Voyager 1 and 2 probes (Yeghikyan 2018 and references therein).

According to Yeghikyan (2018), in a time shorter than the dynamic time of the WR nebulae (about $10^{5}$ years), the part of the particles energetic spectrum in the energy range $1-100 \mathrm{MeV}$ will have quite time to form. It should be emphasized that with the expansion of the nebula, that is, with an increase in its inner radius, its ability to produce energetic particles can be estimated by a semi-analytical relation (Yeghikyan 2018):

$$
\eta \frac{1}{4 \pi} \frac{\dot{M}_{f} V_{f}^{2}}{4 \pi r_{i}^{2}}=\int_{E_{1}}^{E_{\max }} J_{E}(E) d E
$$

where $\dot{M}_{f}$ is the rate of mass loss by the WR star $(\mathrm{g} / \mathrm{s}), V_{f}$ is the wind velocity $(\mathrm{cm} / \mathrm{s}), J_{E}(E)$ is the differential intensity of accelerated particles $\left(\mathrm{erg} \cdot \mathrm{cm}^{-2} \cdot \mathrm{s}^{-1} \cdot \mathrm{sr}^{-1} \cdot \mathrm{MeV}^{-1}\right)$, and $\eta$ is the fraction of kinetic wind energy spent on acceleration of protons by a shock wave in the energy range $E_{1} \leqslant$ $E \leqslant E_{\max }$. By the way, according to the generally accepted theory of the origin of cosmic galactic rays in supernovae expanding shell, usually $\eta=0.1$ - 0.3, but according to later observations and numerical calculations larger values are also possible, $\eta=0.5$ (Ellison et al. 2005). 
It is interesting to compare the estimate (14) for the solar wind with the measurements of the Voyager 1 probe (Yeghikyan, 2018 and references therein). Putting

$$
J_{E}(E)=J_{E}\left(E_{1}\right)\left(\frac{E_{1}}{E}\right)^{\gamma}, J_{p}(E)=\frac{J_{E}(E)}{E_{1}}
$$

where $E_{1}=1 \mathrm{MeV}, \gamma=2-4$, for the differential intensity of particles with $E=$ $E_{1}=1 \mathrm{MeV}$, (in units particles $\cdot \mathrm{cm}^{-2} \mathrm{~s}^{-1} \mathrm{sr}^{-1} \mathrm{MeV}^{-1}$ ) we have (Yeghikyan 2018):

$$
J_{p}\left(E_{1}\right)=\frac{J_{E}\left(E_{1}\right)}{E_{1}}=\frac{\gamma-1}{E_{1}^{2}} \eta \frac{1}{4 \pi} \frac{\dot{M}_{f} V_{f}^{2}}{4 \pi r_{i}^{2}} .
$$

Taking $\dot{M}_{f}=2 \cdot 10^{-14} M_{\odot} / y r, V_{f}=400 \mathrm{~km} / \mathrm{s}, r_{i}=100$ a.u., $\eta=0.1$, for $\gamma=$ 2,3,4 we get, respectively, $J_{p}\left(E_{1}\right) \cong 0.8,1.6,2.4$ particles $\cdot \mathrm{cm}^{-2} \mathrm{~s}^{-1} \mathrm{sr}^{-1} \mathrm{MeV}^{-1}$, which coincides with the Voyager-1 measurements up to a factor of 2, namely, $\approx 1.4$ in the same units (Stone et al. 2005; Scherer et al. 2008). We underline again that the phenomenological estimate (14) gives the same accuracy (within a factor less than 2), if we choose $\eta=0.1$.

We now turn to the calculation of the differential intensity of energetic protons under WR nebular conditions. Taking $\dot{M}_{f}=10^{-5} M_{\odot} / y r$, $V_{f}=1500 \mathrm{~km} / \mathrm{s}, r_{i}=3 \cdot 10^{18} \mathrm{~cm}, \eta=0.1$, then (14) gives values where the concentration of the fast wind at the shock is determined by the relation $n_{1}=\dot{M}_{f} / 4 \pi r_{i}^{2} V_{f} m_{p}$.

It should be emphasized that the average galactic value (for our Galaxy) $J_{p}\left(E_{1}\right)=2 \cdot 10^{-4}$ particles $\cdot \mathrm{cm}^{-2} \mathrm{~s}^{-1} \mathrm{sr}^{-1} \mathrm{MeV}^{-1}$ (Scherer et al. 2008), that is, on the inner boundary of the nebula ( 1 or, say, $5 \mathrm{pc}$ ) intensity of energetic particles which is 1000 or 40 particles $\cdot \mathrm{cm}^{-2} \mathrm{~s}^{-1} \mathrm{sr}^{-1} \mathrm{MeV}^{-1}$, respectively, by more than several orders of magnitude, ( 7 or 5 ) exceeds the mean galactic one. This is not surprising, since even the heliosphere formed by a much weaker wind nevertheless causes the appearance of similar accelerated particles by 4 orders of magnitude greater than intensity of the average galactic one (Scherer et al. 2008).

Thus, WR nebulae can be a very powerful local source of energetic particles (with not very high energies) and it would be interesting to investigate their possible observational manifestations in the nebula itself.

We emphasize that the most interesting from the point of view of energy sources for chemical reactions are particles with energies of $1-10 \mathrm{MeV}$, since even with the minimal observed exponent $(\gamma \approx 2.3)$, the spectral steepness is quite noticeable, and in the integrals describing, for example, the fraction of the absorbed energy, particles with energies greater than $10-20 \mathrm{MeV}$ practically do not contribute. Therefore, when using formulas describing the energy spectra, one should always keep in mind the reservations with which they were obtained, especially in the case of relativistic particles. 
Due to the high values of the fluxes of energetic particles in the WR nebulae, it is interesting to estimate the doses (absorbed energy) of PAHs in these nebulae. We have for the intensity of accelerated particles expression (14), in the energy range of 1-100 MeV, important in the irradiation of different species, as well as in the ionization of $\mathrm{H}_{2}$. Assuming $\dot{M}_{f}=\cdot 10^{-5} M_{\odot} / y r$, $V_{f}=1500 \mathrm{~km} / \mathrm{s}$, for the differential intensity of the energetic protons with $E_{1}=1 \mathrm{MeV}$, one can write for two values of the inner radius $r_{i}=1$ or $5 \mathrm{pc}$ :

$$
J_{p}\left(E_{1}\right)=40-1000 \frac{\text { particles }}{\mathrm{cm}^{2} \cdot \mathrm{s} \cdot \mathrm{sr} \cdot \mathrm{MeV}} .
$$

Thus, at standard values of the parameters of the WR nebulae, the obtained estimates of the intensity at the inner boundary of the nebula are 5-7 orders of magnitude larger than the average galactic one (Scherer et al. 2008, their Fig.2):

$$
J_{p}\left(E_{1}\right)=1.0 \cdot 10^{-4} \frac{\mathrm{particles}}{\mathrm{cm}^{2} \cdot \mathrm{s} \cdot \mathrm{sr} \cdot \mathrm{MeV}} .
$$

The influence of the magnetic field (if present) can be twofold: on the one hand, protons can be reflected from magnetic inhomogeneities, and, depending on the intensity and geometry of the magnetic field $(1-10 \mu \mathrm{G})$, the flux can be decreased by 1 order (Schekinov 2005, and references therein). On the other hand, non-thermal protons of relatively low energies may possibly be accelerated to values of $1-10 \mathrm{MeV}$ in the presence of magnetohydrodynamic turbulence with a specific spectrum (Fermi-2 process) (Hendrix et al. 2016; Monnier et al. 2007). Thus, in this case, the actual particle intensity at the outer edge of the nebula can decrease, at most (taking into account the geometric divergence), by 2 orders of magnitude to $J_{p}\left(E_{1}\right)=0.1-10$ particles $/\left(\mathrm{cm}^{2} \cdot \mathrm{s} \cdot \mathrm{sr} \cdot \mathrm{MeV}\right)$.

It has already been noted above that the distances to the nearest neighbor are calculated for a sphere uniformly filled with stars of the same type, using the obvious formula (13). Since all massive stars of the cluster are considered similar, we will also be interested in half of this distance, that is, $r_{i}=d / 2$.

These values are necessary for calculating the total corpuscular radiation field from the winds of WR stars, because if the flux from one WR star at a distance $r$ is $F_{0} / r^{2}$, then from $N$ such stars in super-association with radius $R_{e f f}$ with uniform filling will be

$$
F \approx \frac{F_{0}}{r^{2}} \frac{N}{\frac{4}{3} \pi R_{\text {eff }}^{3}} \frac{4}{3} \pi r^{3} \approx \frac{N F_{0} r}{R_{\text {eff }}^{3}} \approx \frac{F_{0} r}{d^{3}},
$$

where $F_{0}$ is the flux at a distance of 1 pc from the star. At the boundary, when $r \cong R_{e f f}$, the flux will be determined by the expression

$$
F \approx \frac{N F_{0}}{R_{e f f}^{2}} .
$$


By the formula (20) the lower limit of the flux is determined at any point of the cluster. The method of calculating $F_{0}$ is given above, and now we will explain the need for calculating the total flux. The fact is that the super-associations have dimensions $R_{e f f} \approx 100 \mathrm{pc}$, while the distance to the nearest neighbor is about $d \approx 10 \mathrm{pc}$ (that is $r_{1 / 2}=d / 2 \approx 5 \mathrm{pc}$, see Table 3). When a gas concentration is of about of several $10-100 \mathrm{~cm}^{-3}$, a neutral, $\mathrm{HI}$, and then molecular, $\mathrm{H}_{2}$ layers are formed on sizes $\cong d$ protecting $\mathrm{PAH}$ from hard UV radiation. This can be easily verified by calculating the ionization structure of super-association regions with sizes $\cong d$ using the Cloudy computer model (Ferland et al. 2017). Everywhere, at half the distance of the nearest neighbor $d$, the medium is neutral, or even molecular, and can protect PAHs molecules (Yeghikyan, Martirosyan 2019).

But at densities and sizes about of $R_{e f f}$, the medium of super-association is practically transparent for protons with energies of 1-100 MeV and higher. Such protons are formed at the shock where the stellar wind interacts with the interstellar medium (Yeghikyan 2018). The advantage of formula (20) is that despite its inaccuracy (for use at any point of super-association), it is nevertheless quite suitable for our purposes, namely, determining the lower boundary of the flux to solve the question of the survival of $\mathrm{PAH}$ molecules. Even more accurate relations can be used if we take into account the fact that the given star in the cluster is surrounded by 6 nearest stars at a distance $d$, and at the point $r_{i}=d / 2$ the radiation comes first from two nearest stars and second from 4 stars with distances $\sqrt{5} d / 2$. Thus, the lower boundary value of the flux is estimated, and the inaccuracies associated with the determination of the flux at $r_{i}=1 \mathrm{pc}$ are mutually compensated.

Now it is possible to calculate $d D_{p} / d t$, that is the rate of PAH irradiation by energetic protons in WR nebulae using a simple relation (Yeghikyan 2018, and references therein):

$$
n_{t} \cdot M\left(n_{t}\right) \cdot \frac{d D_{p}}{d t}=\int_{E_{1}}^{E_{2}} F(E) S(E) d E, D_{p}=\frac{d D_{p}}{d t} \cdot t
$$

where $F(E)=4 \pi J_{p}(E)$ and $S(E)=-d E / d x$ is the particle energy loss during the passage of the path $d x$ (in units $\mathrm{keV} / \mu$ ) in the dust particle with the concentration $n_{t}$ and molecular weight $M\left(n_{t}\right)$ (accordingly, $S(E)$ that is the amount of energy absorbed by the dust particle per unit length will be positive). $S(E)$ in the energy range of $1-100 \mathrm{MeV}$ can be easily calculated by the Bethe-Bloch formula, using the SRIM computer program (Ziegler et al. 2003). For PAHs, for example, anthracene, $\mathrm{C}_{14} \mathrm{H}_{10}$, energy losses calculated by SRIM can be found in (Ziegler et al. 2003).

Further, choosing the least steep type of the spectrum, $F(E)=F\left(E_{1}\right)$. $\left(E_{1} / E\right)^{2}$, we obtain $d D_{p} / d t=2.263 \cdot 10^{-11} \mathrm{eV} / \mathrm{s} /$ a.m.u. at a distance of $r_{i}$ $=1 \mathrm{pc}$ from the star. It is possible to calculate the total radiation dose of dust $D_{p}$ for the values of distances $r_{i}=1 \mathrm{pc}$ and $d / 2$, respectively, taking into 
account the (minimal) possible number of nearby stars. In particular, for the characteristic time interval $t=500000$ years at $r_{i}=1 \mathrm{pc}$, the irradiation dose is $D_{p}(1 p c)=\left(d D_{p} / d t\right) \cdot t \cdot\left(1+6 /(d-1)^{2}\right) \mathrm{eV} /$ a.m.u. For larger distances comparable to distances of order $d$, for example, when $r_{i}=d / 2 \mathrm{pc}$, the irradiation dose is $D_{p}(d / 2 p c)=D_{p}(1 p c) \cdot 11.2 /(d-1)^{2} \mathrm{eV} /($ a.m.u. $)$. Values $D_{p}$ are given in Table 3 for the above distances. We emphasize that in the table shown are the lower limits of dose values.

According to experiments (van der Burgt et al. 2018; Dunne et al. 2017), the irradiation of anthracene with low-energy electrons (5-100 eV) leads to its fragmentation, starting from approximately $45 \mathrm{eV}$. It should also be true for protons (including higher energies), and when the corresponding dose is accumulated in the molecule, and for other PAHs (Champeaux et al. 2014; van der Burgt et al. 2018).

With a deficit of hydrogen in the stellar wind, that is, with the prevalence of helium, the resulting value should be multiplied by 10 , since the energy loss of $\alpha$-particles is an order of magnitude greater (Ziegler et al. 2003), and doses can reach $1000 \mathrm{eV} /$ a.m.u. and higher.

\begin{tabular}{|c|c|c|c|c|c|c|}
\hline WR number & $\Sigma N\left(L_{c}\right), s^{-1}$ & $N_{s}$ & $R_{\text {eff }}, \mathrm{cm}$ & $d, p c$ & $D_{p}(1 p c), e V / a . m . u$. & $D_{p}(d / 2), e V / a . m . u$. \\
\hline 31 & $2.31+57$ & $2.310+7$ & $2.199+21$ & 2.50 & 1307 & 6487 \\
\hline 40 & $1.94+54$ & 19420 & $3.408+20$ & 4.11 & 578.8 & 670.7 \\
\hline 48 & $1.62+54$ & 16230 & $2.564+20$ & 3.28 & 768.5 & 1653 \\
\hline 108 & $9.51+53$ & 9508 & $6.331+20$ & 9.69 & 385.5 & 57.23 \\
\hline 190 & $5.81+51$ & 58 & $1.384+20$ & 11.6 & 376.2 & 37.60 \\
\hline 277 & $2.32+53$ & 2321 & $3.268+20$ & 8.00 & 400.8 & 91.65 \\
\hline 288 & $3.03+53$ & 3034 & $3.237+20$ & 7.25 & 412.0 & 118.3 \\
\hline 292 & $5.05+54$ & 50541 & $4.884+20$ & 4.28 & 556.1 & 578.5 \\
\hline 296 & $3.25+51$ & 32 & $3.136+20$ & 32.0 & 359.3 & 4.183 \\
\hline
\end{tabular}

Table 3: Dose rates and doses of PAH molecules in 500000 years

We should bear in mind the fact that these values can be at least doubled, since radiation from the previous stage $\mathrm{O}$ of the star, whose power is an order of magnitude less $\left(\dot{M}_{f}=10^{-6} M_{\odot} / y r\right)$, but the duration is ten times more $(\mathrm{t}=5$ million years). Thus, everywhere in the WR nebulae PAHs receive the dose of more than 50-100 eV and should have been destroyed. Galaxy N 296 probably is an exception, but of course with such galaxies (and of course, N 31) one should be dealt separately.

Thus, the PAH molecules should have disintegrated in a time less than the lifetime of the cluster. This leads to the conclusion that a permanent source of PAH molecules exists in a nebula associated with a cluster of young and very massive stars. Most likely, these PAH molecules are formed in supernova remnants in the same cluster.

The origin of dust, which is observed in WR nebulae, is still under discussion (Hendrix et al. 2016), it is possible that dust (and PAH) is produced under conditions of the colliding winds of massive WR pairs (Hendrix et al. 2016), but in the case of PAHs, as we found out, this is impossible due to 
inevitable destruction during the star lifetime.

\section{Conclusion}

In this paper, we analyzed the conditions in WR galaxies, and for 9 of them the numbers of hydrogen ionizing photons were calculated based on the observed fluxes corrected for interstellar extinction. Then, the effective volume and effective radius radiation of nebulae were calculated, and assuming the number of massive stars in the cluster was equal to the number of WR stars, and, knowing also the theoretical number of hydrogen-ionizing photons from one star, the numbers of WR stars in each cluster were calculated. Further, the distance to the nearest neighbor (with a uniform distribution) turned out to be of the order of several parsecs, and besides based on the theoretical models calculated by us the gaseous medium at such a distance is already neutral, and even molecular completely protecting the nebula from UV radiation. However, the medium is exposed not only to electromagnetic, but also to corpuscular irradiation from protons with energies of 1-100 MeV, which arise in the processes of diffuse acceleration at the shock in the Fermi-1 process. The accumulated doses of radiation from these protons, calculated during the lifetime of the super-association, were everywhere in the nebula more than $50-100 \mathrm{eV}$, which should lead to the destruction of PAHs. However, PAHs are observed in WR galaxies, therefore, we came to the conclusion that PAH sources are permanently exist, most likely in supernova remnants.

\section{References}

Ambartsumian, V. 1964, Superassociations in distant galaxies, ed. F.J.Kerr and A.W.Rodgers, IAU Symp. 20, p. 122

Brinchmann, J.; D. Kunth, D.; Durret, F. 2008, A\&A, 485, 657

Champeaux, J.-P.; Moretto-Capelle, P.; Cafarelli, P.; Deville, C.; Sence, M.; Casta, R. 2014, MNRAS, 441, 1479

Conti, P. Ap.J., 1991, 377, 115

Crowther, P. 2007, ARAA, 45, 177

Crowther, P.; Hadfield, L.; Schild, H.; Schmutz, W.; de Grijs, R.; Gonzalez, R. M.; Decourchelle, A. 2008, Mem. S.A.It., 79, 44, 2008

Delgado (eds.), Starbursts, 2005, Springer, 21

Drury, L. 1983, Rep. Prog. Phys., 46, 973

Dunne, M., Gradziel, M., van der Burgt, P., 2017, Journal of Physics: Conf. Series 875, 062013

Ellison, D., Decourchelle, A., Ballet, J. 2005, A\&A, 429, 569

Ferland, G.; Chatzikos, M.; Guzman, F.; Lukins, M. et al. 2017, RMAA, 53,385 
Hamann, W.-R.; Gräfener, G.; Liermann, A. 2006, A\&A 457, 1015

Hendrix, T., Keppens, R., van Marle, A., Camps, P., Baes, M., Zakaria, M. 2016, MNRAS, 460, 3975

Martirosyan, J.; Sargsyan, L. 2012, Astrophysics, 55, 306

Meynet, G.; Maeder, A.; 2005, A\&A, 429, 581

Monnier, J., Tuthill, P., Danchi, W., Murphy, N., Harries, T. 2007, Astrophys. J. 655, 1033

Risaliti, G.; Maiolino, R.; Salvati, M. 1999, Astrophys. J., 522, 157

Shchekinov, Y. 2005, Astron. Rep. 49, 269, 2005

Scherer, K., Fichtner, H., Ferreira, S., Büsching, I., Potgieter, M. 2008, ApJ., 680, L105.

Stone, E., Cummings, A., McDonald, F., Heikkila, B., La, N., Webber, W. 2005, Science, 309, 2017

van der Burgt, P., Dunne, M., Gradziel, M. 2018, The Europian Physical Journal D, 72,31

Vink1, J.; Gräfener, G.; Harries, T. 2011, A\&A, 536, L10

Yeghikyan, A. 2018, Astrophys., 61, 469

Yeghikyan, A.; Martirosyan, J. 2019, Astrophysics, to be submitted

Ziegler, J.; Biersack, J.; Littmark, U. 2003, The Stopping and Range of Ions in solids, Pergamon Press, NY, 2003. 